\title{
La formación y la gestión del talento en las empresas más valoradas en recursos humanos en España
}

\author{
Training and talent management of the most valued \\ human resources companies in Spain
}

\author{
Francisco Jesús Ferreiro-Seoane ${ }^{1 *}$, Manuel Octavio Del Campo Villares ${ }^{2}$, \\ Marta Camino-Santos ${ }^{1}$ \\ ${ }^{I}$ Universidad de Santiago de Compostela, España \\ ${ }^{2}$ Universidad de A Coruña, España.
}

Recibido el 25 de agosto de 2017; aceptado el 1 de octubre de 2018

Disponible en Internet el: 4 de diciembre de 2018

\section{Resumen}

Este artículo pretende conocer el perfil de las empresas más atractivas para el desempeño profesional en España a través de las variables Formación y Gestión del Talento, en relación con otras variables objetivas como: actividad económica; nacionalidad; ubicación geográfica; tamaño y cotización en bolsa. Las técnicas de análisis estadístico empleadas han sido: regresiones lineales múltiples mediante mínimos cuadrados ordinarios, correlaciones de Pearson, varianzas unifactoriales con el test de Levene, promedios y ponderaciones. No se obtiene un perfil único para ambas variables. Por un lado, la Formación alcanza mayores valores en actividades sanitarias en las empresas del área mediterránea ubicadas en la zona norte de España; y son grandes organizaciones que cotizan en el mercado bursátil; son estas dos últimas variables relevantes estadísticamente. En cambio, en la Gestión del Talento los mayores valores se encuentran en el sector profesional, científico y técnico, en las empresas anglosajonas ubicadas en el centro de España, en la que predominan las grandes compañías que cotizan en bolsa; esta última variable es relevante estadísticamente.

Código JEL: J24, J28

Palabras clave: Formación; Gestión del talento; Empresas; Recursos humanos; España

\footnotetext{
*Autor para correspondencia.

Correo electrónico: franciscojesus.ferreiro@usc.es (F. J. Ferreiro-Seoane)

La revisión por pares es responsabilidad de la Universidad Nacional Autónoma de México.
} 


\begin{abstract}
The objective of this article is to know the profile of the most attractive companies for professional development in Spain through the Training and Talent Management variables, in relation to other objective variables such as: economic activity, nationality, geographical location, size, and stock market listing. The statistical analysis techniques used are multiple linear regressions through ordinary least squares, Pearson correlations, unifactorial variance with Levene's test, averages, and weightings. A unique profile is not obtained for both variables. On the one hand, Training obtains greater values in sanitary activities in companies from the Mediterranean located in the area north of Spain, and they are also large organization listed in the stock market; it is these two variables that are statistically relevant. On the other hand, Talent Management has greater values in the professional, scientific, and technical sector, in Anglo-Saxon companies located in the center of Spain, with large companies listed in the stock market predominating; it is this last variable that is statistically relevant.
\end{abstract}

Código JEL: J01, J08, J24, J28

Keywords: Training; Talent management; Companies; Human resources; Spain

\title{
Introducción
}

El talento y el conocimiento humano han adquirido una importancia vital en todo tipo de organizaciones; el Capital Humano cualificado es actualmente aquel que diseña estrategias, desarrolla los proyectos, marca los objetivos y dirige para alcanzarlos, una empresa funciona con personas para atender necesidades de personas. Por ello, la organización exitosa será aquella, capaz de convertir el conocimiento en información útil y transmitirla a sus clientes, proveedores, accionistas y a todos los integrantes de la organización, de modo que influye positivamente en el entorno y lidera el mercado.

La Revista Actualidad Económica (RAE) publica anualmente un ranking con las cien empresas más atractivas para el desempeño laboral en España, el cual se obtiene a través de la medición de seis variables independientes: la formación; gestión del talento; responsabilidad social corporativa (RSC); retribución y compensación; ambiente de trabajo; percepción de los empleados de la empresa, y una valoración total, que es una variable dependiente de las anteriores.

El objetivo del presente artículo es analizar el ranking de las 100 empresas más valoradas para trabajar para el período 2013-2016, publicado por la RAE, a través de las variables Formación y Gestión del Talento, y su relación con otras variables objetivas como la actividad económica de las empresas, la nacionalidad, la ubicación de la sede central en el territorio, el tamaño de la empresa y si cotizan en el mercado bursátil. 


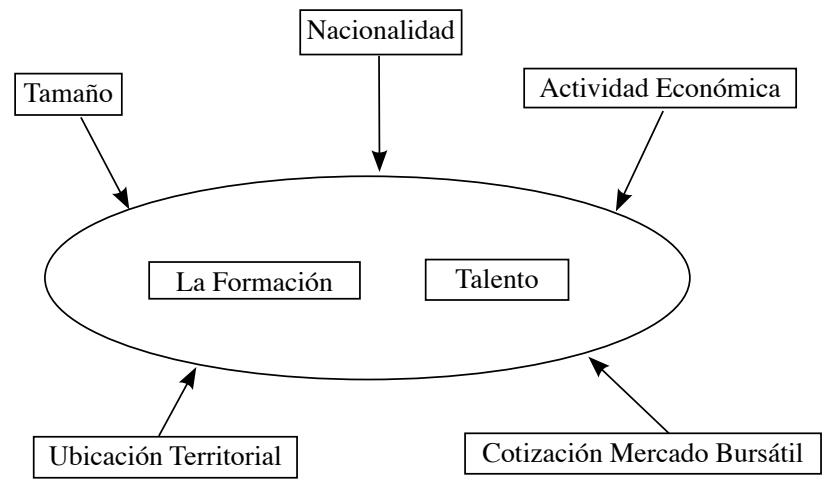

Figura 1.

Análisis de la Formación y Gestión del Talento y su relación con otros factores.

Fuente: Elaboración propia

Para alcanzar dichos objetivos se hace una revisión de la literatura. Posteriormente se define la metodología empleada, se identifican las variables objeto de estudio y su justificación, el tamaño muestral y las técnicas de análisis empleadas. A continuación, se procede al análisis cuantitativo y sus resultados, se exponen las limitaciones encontradas, para finalizar con el apartado de discusión y conclusiones.

\section{Marco teórico}

En un mundo globalizado y competitivo como el actual, la consideración de mejores empresas para el desempeño profesional adquiere una relevancia especial, las cuales según Friedman (2014) y Morgan (2014) dependen de nuevas conductas, la tecnología, el grado de movilidad y el nivel de globalización alcanzado. Actualmente, los profesionales no limitan sus carreras profesionales a una sola empresa, pues hay una mayor movilidad geográfica (Guest, 2007, pp. 128 y ss.). En la actualidad, los incentivos tradicionales como el salario, no son los únicos que determinan las empresas más atractivas para el desarrollo profesional.

Se aprecia en las dos últimas décadas como una de las herramientas del branding de los empleadores (Joo y McLean, 2006) es la difusión de su posición en alguno de los rankings de las empresas más atractivas para trabajar (con tendencia a fijar 100). El objetivo es "emitir una señal" para atraer y retener a un capital humano que se considera como el recurso más valioso de una organización (Joyce, 2003); Del Campo y Salcines (2008). Este hecho es reconocido en las publicaciones de Hall (1992) y Bonache (1996) que afirmaban que uno de los elementos intangibles fundamentales para la organización son los recursos humanos. Wright, McMahan y McWilliams, (1994) lo definen como el "conjunto de capital humano bajo el control de la empresa en una relación directa de empleo". Así, las propias empresas intentan atraer y retener al talento, hecho que se refuerza con la presencia en alguno de los rankings como una de las mejores empresas para trabajar (Lenaghan y Eisner, 2006, pp. 99 y ss.).

Entre tales rankings, se encuentra el Fortune 100 Best Companies to Work for. Glassdoor que publica anualmente para Estados Unidos y algunos países de Europa, listas de Best Places to Work. Marcaempleo la cual edita su ranking Merco Talento, que ordena por puntuación 
a las "100 mejores empresas españolas para trabajar". Universum, elabora rankings de "top employers" para varios países. El Top Employers Institute publica, un listado de empresas certificadas por ellos por "crear condiciones óptimas para el desarrollo de sus empleados, tanto personal como profesionalmente". Por su parte, la revista Workforce elabora un ranking de The World's Top Companies for HR mediante la combinación de varios índices o rankings, entre ellos, el de Great Place to Work.

Fulmer, Gerhart y Scott, 2003; Romero, (2004), resaltan cómo la presencia en algún ranking de las empresas más atractivas para el personal influye sobre las condiciones más idóneas de las mismas para trabajar. Hinkin y Tracey, (2010) destacan que estar en algún ranking convierten a las empresas en las mejores en sus prácticas de recursos humanos. Según Ballou, Godwin y Shortridge (2003) afecta a las actitudes de los empleados hacia el puesto de trabajo. Investigadores españoles (Guinot, Chiva y Mallén, 2015), en su trabajo sobre capacidad de aprendizaje organizativo, han usado para seleccionar sus casos varios rankings de las mejores empresas para trabajar en España, entre ellos el de $R A E$.

El objeto de este artículo se asienta en dos parámetros: la Formación y la Gestión del Talento. Así, Finegold y Sosckice (1988); Betcherman et al. (1997) y Del Campo y Salcines (2008) concluyen que la toma de decisiones sobre formación es clave en el resultado empresarial y debe ser tenida en cuenta por los directivos. Según Smith (1993) y Osterman (1994) la formación es esencial para la supervivencia de la empresa. La Teoría del Capital Humano de Mincer (1962) argumenta que la educación y la formación son dos formas de inversión complementarias, siendo la formación una inversión rentable (OCDE, 1991).

Existen evidencias que indican que la excelencia de la organización solo puede alcanzarse si se introducen intervenciones de formación junto con otros factores sociales, (Hosie et al., 2013). Además, los empleados satisfechos tienen mayor devoción por su trabajo y son más productivos, (Nijhof et al., 1998; Baron, 1991).

\section{Método}

\section{Variables}

La elaboración del ranking de las 100 empresas más atractivas para el desempeño laboral en España se efectúa a través de seis variables independientes y una dependiente (tabla 1), cuya ponderación potencial varía según la variable en cuestión y puede alcanzar un máximo de 1.000 puntos. El estudio se centra en dos de las variables independientes: Formación y Gestión del Talento, por estar vinculadas de forma directa al conocimiento. Además, son las más relevantes desde un punto de vista cuantitativo pues alcanzan un valor potencial del $46 \%$ de la variable dependiente total del valor. 
Tabla 1

Variables para valorar las organizaciones más atractivas para el desempeño profesional en España.

\begin{tabular}{llll}
\hline Variable & Descripción & Puntos & $\%$ s /total \\
\hline La Formación & Valora la inversión recibida por el empleado & 220 & $22.0 \%$ \\
La Gestión del Talento & $\begin{array}{l}\text { Aborda la proyección, el rendimiento y la } \\
\text { rotación no deseada }\end{array}$ & 240 & $24.0 \%$ \\
$\begin{array}{l}\text { Resto de variables: Retribución y Compensación; Ambiente de Trabajo; } \\
\text { Responsabilidad Social Corporativa (RSC); Valoración de Empleados. }\end{array}$ & 540 & $54.0 \%$ \\
& $\begin{array}{l}\text { Corresponde a la suma de las anteriores } \\
\text { variables }\end{array}$ & 1000 & $100.0 \%$ \\
\hline
\end{tabular}

Fuente: Elaboración propia a partir de los datos publicados en Actualidad Económica 2013-2016.

Para Gallie (2009,pp.6) el concepto de calidad del trabajo radica en el nivel de competencias, el grado de autonomía, discreción en las tareas, las oportunidades para el desarrollo de habilidades, la seguridad en el trabajo y el grado de compatibilidad con el equilibrio trabajofamilia.

Grimshaw y Rubery (2007), Fields, Chan y Akhtar (2002) y Jackson y Schuler (1995), enfatizan en el papel de los factores contextuales (mercados, políticos, institucionales, entorno social, cultura) para explicar las diferencias en las prácticas y los resultados de la gestión de recursos humanos entre empresa. La literatura reciente pone de relieve la relación entre la estandarización universal de prácticas y el ajuste al contexto local (Amossé et al., 2016; Gallie, 2007; Hodulak, 2017; Ibrahim y Shah, 2013; Quintanilla y Ferner, 2003).

El escaso esfuerzo de construcción de teoría sobre los rankings de las mejores empresas para trabajar (Joo y McLean, 2006) se extiende a la ausencia de una teoría específica sobre la asociación entre las puntuaciones obtenidas por las empresas en esos rankings y los valores de otros factores organizativos, económicos, culturales o de otra naturaleza. Por ello, se han seleccionado los factores explicativos a partir de investigaciones empíricas dedicadas a las prácticas de gestión de recursos humanos, en ocasiones únicamente dirigidas a la detección de los efectos de alguno o algunos de los factores de interés.

Actividad económica. Jackson y Schuler (1995, pp. 251 y ss.) incluyen, entre los factores contextuales influyentes en las prácticas de recursos humanos, las características del sector de actividad. Según Conway et al., 2008 se pueden categorizar de varios modos: servicios / industria.... En este estudio se clasifican las empresas según el sector de actividad para lo que se usa la Clasificación Nacional de Actividades Económicas (CNAE).

Nacionalidad. A pesar de que Ibrahim y Shah (2013) no encuentran efectos de la nación de origen en las prácticas de recursos humanos en empresas malayas; Ferner (1997) presentó una relación de las diferencias sistemáticas en la gestión de recursos humanos en las empresas multinacionales en función del país de origen. Liu (2004) y Guthrie et al. (2008) documentan los hallazgos en el mismo sentido con investigaciones empíricas más recientes. En esta investigación se identifica cada empresa con el país de origen, lo que nos permitirá obtener estadísticas agrupadas por nacionalidad de las variables objeto de estudio. A su vez, se han 
agregado en áreas geo-culturales tales como países Anglosajones, Centro y Norte de Europa, Mediterráneo y resto; lo que permite una visión más global de esta dimensión.

Ubicación territorial. En algunos trabajos se encuentran ciertas diferencias entre las prácticas de recursos humanos en distintos países detectadas por investigaciones comparativas que emplean diversas técnicas econométricas (Amossé et al., 2016; Conway et al., 2008; Fields, Chan y Akhtar, 2002; Grimshaw y Rubery, 2007; Paawee y Boselie, 2007). Uno de los propósitos de esta investigación es averiguar si la ubicación específica en un territorio tiene influencia en las variables Formación y Gestión del Talento. Por este motivo, se clasificaron todas las empresas según la situación regional (Municipio, Provincia y Comunidades Autónomas) de la sede central en España. Los análisis se harán a través de la dimensión regional de las Comunidades Autónomas, agrupadas además en áreas culturales tales como: Centro, Mediterráneo, Norte de España y resto. Esto nos permitirá hacer estudios a diversos niveles de escala regional.

Tamaño de la empresa. El tamaño es uno de los factores potencialmente más influyentes en las prácticas de recursos humanos (Fields, Chan y Akhtar, 2002, p. 265 y ss.), en cambio no parece haber consenso sobre el signo, positivo o negativo, de sus efectos. Por un lado, Kortekaas (2007), halla un notable impacto de signo positivo del tamaño pequeño/medio en las conductas de los empleados (incluidos el compromiso o la satisfacción en el trabajo) y un impacto de signo negativo del mismo tamaño en un indicador de rendimiento operacional (absentismo y bajas por enfermedad). Kok y Uhlaner (2001) hallan una asociación entre el aumento del tamaño de las empresas y la formalización de las prácticas de recursos humanos. Morgan (2014) sugiere que las empresas pequeñas tienen varias ventajas como la flexibilidad de roles, relación empresario vs trabajador cercana, funciones, y estilos de trabajo para los empleados, entre otros. En cambio, Ibrahim y Shah (2013, pp. 7, 14 y ss.) aducen que las empresas pequeñas carecen de los recursos necesarios para adoptar prácticas de gestión de recursos humanos progresivas. En este artículo la medida usada será el número de trabajadores que tiene dicha organización en España.

Cotización. Conway et al. (2008, pp. 638) concluyen que la investigación sobre sus efectos no generó conclusiones claras. Los resultados empíricos hallados en el Reino Unido muestran que la cotización se asocia positivamente con el trabajo en equipo y la remuneración relacionada con el rendimiento. En el caso de Francia la cotización se asocia con la autonomía y la formación del trabajador. Con esta variable se tratará de analizar si es relevante la cotización o valor de mercado de las empresas que figuran en el ranking de la $R A E$.

\section{Muestra y técnicas de análisis}

El tamaño muestral corresponde a las cien empresas más atractivas del ranking publicado cada año por la $R A E$ para el período 2013-2016, lo que suponen 400 observaciones y 182 empresas, ya que algunas de ellas se repiten en todos o algunos de los años, lo que hace un ratio de 2.2 del número de observaciones por empresa. De las 182 empresas, 40 repiten presencia los cuatro años, lo que representa sólo el $21.9 \%, 33$ empresas están presentes en tres años (18.13\%), las 32 empresas con presencia en dos años representan el 17.5\%, y las 77 empresas en sólo un año suponen el $42.3 \%$, por lo que se aprecia una continuada entrada y salida de empresas en el ranking de la $R A E$. 
El proceso de elaboración del ranking ha consistido en la entrega de un cuestionario con un centenar de preguntas por parte de la $R A E$ a los departamentos de Recursos Humanos de las empresas que llevan más de cinco años en España y que hayan tenido una plantilla superior a los 100 empleados en el período tratado. Una vez las empresas hayan respondido a dichos cuestionarios, expertos consultores y profesionales de Recursos Humanos independientes de la RAE procedieron a la medida y validación del análisis del ranking y posterior publicación con la valoración para cada empresa de las variables: formación; gestión del talento; responsabilidad social corporativa (RSC); retribución y compensación; ambiente de trabajo; percepción de los empleados de la empresa, y una valoración total.

Así es que, para alcanzar nuestro objetivo, al ranking empleado se le han agregado para cada empresa nuevas variables como la nacionalidad, ubicación, actividad de la empresa, tamaño y si cotiza en el mercado bursátil. El objetivo es analizar si estas variables agregadas influyen o no en el valor obtenido en formación y gestión del talento por las empresas y conocer mejor su perfil.

Como técnicas de análisis se usarán: regresiones lineales múltiples mediante mínimos cuadrados ordinarios para cada uno de los cuatro años, correlaciones de Pearson, varianzas unifactoriales con el test de Levene y estadísticos que permitan medir la Formación y Gestión del Talento de las empresas en número, porcentajes y valores promedios.

\section{Análisis y resultados}

\section{La formación y la gestión del talento según la actividad económica de las empresas}

En la tabla 2 se puede apreciar el valor de la formación de las empresas clasificadas por sectores económicos. La primera posición es ocupada por las actividades sanitarias y servicios sociales, las cuales alcanzan una valoración promedio de 183.3 puntos. En segunda posición se sitúan las actividades profesionales, científicas y técnicas con 182,2 puntos y donde se encuentran empresas de consultoría internacional como Deloitte, PWC o KPMG. Destacar que un sector tan relacionado con el conocimiento como el de la educación se sitúe en novena posición con poca representatividad en el número de empresas.

Si se centra la atención en la variable Talento, las diferencias respecto al caso anterior son notables. En primer lugar, decir que, la correlación de Pearson con la Formación en función de los valores promedios obtenidos por sectores es de tan sólo el 12.2\%. Aquí se observa cómo el sector que ocupa la primera posición es el de las actividades profesionales, científicas y técnicas. De nuevo destaca el sector de educación el cual se sitúa, en la última posición con 127.5 puntos. 
Tabla 2

La Formación y la Gestión del Talento de las empresas por actividades económicas

\begin{tabular}{|c|c|c|c|c|c|c|c|}
\hline Sectores & $\begin{array}{l}\text { Valor promedio } \\
\text { Formación }\end{array}$ & $\begin{array}{l}\text { Posición } \\
\text { Formación }\end{array}$ & $\begin{array}{l}\text { Valor } \\
\text { promedio } \\
\text { Talento }\end{array}$ & $\begin{array}{l}\text { Posición } \\
\text { Talento }\end{array}$ & $\begin{array}{l}\text { Valor } \\
\text { promedio } \\
\text { Total }\end{array}$ & $\begin{array}{l}\text { Posición } \\
\text { Total }\end{array}$ & $\begin{array}{c}\mathrm{N}^{\circ} \\
\text { Empresas }\end{array}$ \\
\hline $\begin{array}{l}\text { Actividades } \\
\text { sanitarias y de } \\
\text { servicios sociales }\end{array}$ & 183.3 & 1 & 170.7 & 11 & 783.6 & 1 & 7 \\
\hline $\begin{array}{l}\text { Actividades } \\
\text { profesionales, } \\
\text { científicas y técnicas }\end{array}$ & 182.2 & 2 & 188.5 & 1 & 779.7 & 4 & 65 \\
\hline Hostelería & 181.7 & 3 & 176.1 & 6 & 746.4 & 8 & 9 \\
\hline $\begin{array}{l}\text { Suministro de } \\
\text { energía eléctrica, } \\
\text { gas, vapor y aire } \\
\text { acondicionado }\end{array}$ & 181.4 & 4 & 162.1 & 14 & 782.5 & 2 & 21 \\
\hline Construcción & 180.7 & 5 & 179.3 & 3 & 743.1 & 9 & 7 \\
\hline $\begin{array}{l}\text { Transporte y } \\
\text { almacenamiento }\end{array}$ & 179.1 & 6 & 175.3 & 7 & 731.7 & 11 & 7 \\
\hline $\begin{array}{l}\text { Industria } \\
\text { manufacturera }\end{array}$ & 176.9 & 7 & 171.8 & 10 & 771.5 & 5 & 47 \\
\hline $\begin{array}{l}\text { Actividades } \\
\text { financieras y de } \\
\text { seguros }\end{array}$ & 176.0 & 8 & 175.0 & 8 & 781.3 & 3 & 97 \\
\hline Educación & 172.5 & 9 & 127.5 & 15 & 672.0 & 14 & 2 \\
\hline $\begin{array}{l}\text { Actividades } \\
\text { administrativas y } \\
\text { servicios auxiliares }\end{array}$ & 170.9 & 10 & 179.8 & 2 & 737.3 & 10 & 23 \\
\hline $\begin{array}{l}\text { Información y } \\
\text { Comunicación }\end{array}$ & 169.0 & 11 & 176.8 & 5 & 755.5 & 6 & 49 \\
\hline $\begin{array}{l}\text { Actividades } \\
\text { inmobiliarias }\end{array}$ & 168.6 & 12 & 177.1 & 4 & 729.4 & 12 & 7 \\
\hline $\begin{array}{l}\text { Comercio al por } \\
\text { mayor y al por } \\
\text { menor }\end{array}$ & 168,1 & 13 & 174.8 & 9 & 751.9 & 7 & 57 \\
\hline $\begin{array}{l}\text { Administración } \\
\text { pública y defensa }\end{array}$ & 160.0 & 14 & 165.0 & 13 & 645.0 & 15 & 1 \\
\hline $\begin{array}{l}\text { Agricultura, } \\
\text { ganadería, } \\
\text { silvicultura y pesca }\end{array}$ & 135.0 & 15 & 170.0 & 12 & 715.0 & 13 & 1 \\
\hline Total general & 175.2 & & 176.7 & & 765.8 & & 400 \\
\hline $\begin{array}{l}\text { Correlación de } \\
\text { Pearson Formación- } \\
\text { Talento }\end{array}$ & & & $12,2 \%$ & & & & \\
\hline
\end{tabular}

Fuente: Elaboración propia a partir de los datos publicados en Actualidad Económica 2013-2016.

\section{La formación y el talento según la nacionalidad de las empresas}

En la tabla 3 se pueden observar los valores de la variable Formación agrupada por países, donde las empresas de Corea del Sur ocupan la primera posición del ranking, con un valor de 188.8 puntos obtenidos con la empresa electrónica LG. La segunda posición es alcanzada por las empresas de nacionalidad irlandesa, las cuales obtienen una puntuación de $187.5 \mathrm{y}$ donde destaca la consultora Accenture. Tanto Corea del Sur como Irlanda presentan un tamaño muestral insuficiente. En tercera posición, se sitúan las empresas suizas, tales como seguros 
Zurich o la farmacéutica Roche Farma, entre otras. Por ello, si se centra el foco de atención en países con una presencia significativa de empresas, Suiza ocupa la tercera posición con 183,4 puntos representada por 14 observaciones; Francia en quinta posición, tiene una valoración media de 178.4 puntos con 34 referencias; le sigue EEUU con 177.2 puntos y 65 compañías y España que es el país que más empresas presenta (155) con 176.4 puntos ocupa la séptima posición. El Reino Unido ocupa la tercera posición en número de empresas, en cambio la valoración media de la Formación la sitúa en la décima posición y a Alemania en la duodécima.

En cuanto a la valoración del talento destacan las empresas de Finlandia y Corea del Sur, aunque ambos países carentes de representatividad en el número de empresas. En tercer lugar, se sitúan las suizas, con una puntuación muy similar a la obtenida en el análisis de la Formación 183.2 puntos. Las empresas del Reino Unido con una baja valoración en el promedio de Formación obtienen la cuarta posición en Gestión del Talento. En el caso de España, destaca el hecho de que pasa de la séptima posición en Formación a la duodécima en Gestión del Talento, lo que revela que ambos factores no siempre van en la misma dirección o que, el tratamiento del capital humano en ocasiones no es el debido. También cabe destacar que las empresas de procedencia portuguesa son de las más valoradas en cuanto a Formación (cuarto puesto) y sin embargo, a la hora de analizar el Talento y la valoración total resultan de las peor consideradas, pues ocupan la posición decimosexta y decimotercera, respectivamente. Esto explica que la correlación entre la Formación y Talento agrupada por países sea del $43.4 \%$.

Tabla 3

La Formación y la Gestión del Talento de las empresas por nacionalidad

\begin{tabular}{|c|c|c|c|c|c|c|c|}
\hline País & $\begin{array}{l}\text { Valor promedio } \\
\text { Formación }\end{array}$ & $\begin{array}{l}\text { Posición } \\
\text { Formación }\end{array}$ & $\begin{array}{l}\text { Valor } \\
\text { promedio } \\
\text { Talento }\end{array}$ & $\begin{array}{l}\text { Posición } \\
\text { Talento }\end{array}$ & $\begin{array}{l}\text { Valor } \\
\text { promedio } \\
\text { Total }\end{array}$ & $\begin{array}{l}\text { Posición } \\
\text { Total }\end{array}$ & $\begin{array}{l}\mathrm{N}^{\circ} \\
\text { Empresas }\end{array}$ \\
\hline Corea del Sur & 188.8 & 1 & 191.3 & 2 & 828.8 & 1 & 4 \\
\hline Irlanda & 187.5 & 2 & 175.0 & 9 & 789.0 & 3 & 4 \\
\hline Suiza & 183.4 & 3 & 183.2 & 3 & 799.9 & 2 & 14 \\
\hline Portugal & 182.5 & 4 & 145.0 & 16 & 731.5 & 13 & 4 \\
\hline Francia & 178.4 & 5 & 177.5 & 7 & 759.1 & 12 & 34 \\
\hline EEUU & 177.2 & 6 & 182.7 & 5 & 773.9 & 6 & 65 \\
\hline España & 176.4 & 7 & 174.2 & 12 & 767.2 & 7 & 155 \\
\hline Países Bajos & 174.3 & 8 & 178.3 & 6 & 761.1 & 10 & 21 \\
\hline Japón & 173.3 & 9 & 175.0 & 10 & 777.3 & 4 & 4 \\
\hline Reino Unido & 171.9 & 10 & 182.9 & 4 & 765.6 & 8 & 40 \\
\hline Suecia & 170.0 & 11 & 169.6 & 14 & 721.2 & 15 & 12 \\
\hline Alemania & 168.8 & 12 & 175.0 & 11 & 760.3 & 11 & 28 \\
\hline Italia & 165.6 & 13 & 167.8 & 15 & 761.8 & 9 & 9 \\
\hline Finlandia & 165.0 & 14 & 192.5 & 1 & 775.0 & 5 & 2 \\
\hline Luxemburgo & 150.0 & 15 & 170.0 & 13 & 722.0 & 14 & 1 \\
\hline Dinamarca & 137.5 & 16 & 120.0 & 17 & 634.5 & 17 & 2 \\
\hline $\begin{array}{l}\text { China } \\
130.0 \\
17\end{array}$ & & & 175.0 & 8 & 650.0 & 16 & 1 \\
\hline Total general & 175.2 & & 176.7 & & 765.8 & & 400 \\
\hline $\begin{array}{l}\text { Correlación Pearson } \\
\text { Formación-Talento }\end{array}$ & & & $43,4 \%$ & & & & \\
\hline
\end{tabular}

Fuente: Elaboración propia a partir de los datos publicados en Actualidad Económica 2013-2016. 
En el análisis por áreas internacionales, recogido en la tabla 4, las empresas de Europa Mediterránea ocupan el primer lugar en Formación y el último en Gestión del Talento, lo que de nuevo muestra la baja correlación entre ambas variables. Otro aspecto destacable es la baja valoración de las empresas de Europa Centro Norte, con países como Dinamarca, Luxemburgo, Finlandia o Suecia. Las empresas de los países anglosajones son las que mantienen posiciones más constantes.

Tabla 4

La Formación y la Gestión del Talento de las empresas por áreas internacionales

\begin{tabular}{llllllll}
\hline Áreas Internacionales & $\begin{array}{l}\text { Valor } \\
\text { promedio } \\
\text { Formación }\end{array}$ & $\begin{array}{l}\text { Posición } \\
\text { Formación }\end{array}$ & $\begin{array}{l}\text { Valor } \\
\text { promedio } \\
\text { Talento }\end{array}$ & $\begin{array}{l}\text { Posición } \\
\text { Talento }\end{array}$ & $\begin{array}{l}\text { Valor } \\
\text { promedio } \\
\text { Total }\end{array}$ & $\begin{array}{l}\text { Posición } \\
\text { Total }\end{array}$ & $\begin{array}{l}\mathrm{N}^{\mathrm{o}} \\
\text { Empresas }\end{array}$ \\
\hline Europa Mediterránea & 176.4 & 1 & 173.9 & 4 & 764.9 & 3 & 202 \\
Anglosajones & 175.6 & 2 & 182.5 & 1 & 771.4 & 2 & 109 \\
Resto & 175.3 & 3 & 182.2 & 2 & 786.0 & 1 & 9 \\
$\begin{array}{l}\text { Europa Centro - } \\
\text { Norte }\end{array}$ & 171.8 & 4 & 175.5 & 3 & 758.3 & 4 & 80 \\
Total general & 175.2 & & 176.7 & & 765.8 & & 400 \\
\hline
\end{tabular}

Fuente: Elaboración propia a partir de los datos publicados en Actualidad Económica 2013-2016.

\section{La formación y la gestión del talento según ubicación territorial de las empresas}

En la tabla 5 se puede apreciar que respecto a la variable Formación, como Cantabria es la Comunidad Autónoma que ocupa la primera posición en el ranking con 184.2 puntos y en la que se sitúan empresas como el Banco Santander, uno de los mayores bancos de Europa. El segundo puesto corresponde a las organizaciones domiciliadas en las Islas Baleares, donde destacan empresas relacionadas con el turismo, al ser ésta una de las zonas con mayor índice turístico del mundo, que alcanzan un total de 181.7 puntos. Casi dos puntos por debajo, en tercera posición aparece el País Vasco, el cual acoge en su territorio a la financiera BBVA (otro de los grandes bancos europeos) y grandes empresas energéticas. Si se presta atención a las comunidades con mayor presencia tenemos a Cataluña con 51 empresas, que ocupa el quinto lugar, y Madrid es la zona con mayor número de empresas con el $76.8 \%$ del total.

En el caso de la variable talento, Galicia es la comunidad con mayor valoración promedio, pues se sitúa con 188.3 puntos en el primer puesto y donde destaca la textil Inditex, una de las empresas con mayor valor del mundo, seguida de las Islas Canarias y Baleares. Como se observa, cambian las posiciones relativas de las comunidades al clasificarlas según Formación o Gestión del Talento, lo que explica que la correlación de Pearson entre ambas variables sea del $23.8 \%$, mucho más baja que por países, aunque más alta que por actividades. 
Tabla 5

La Formación y la Gestión del Talento clasificados según la sede social

\begin{tabular}{llllllll}
\hline CCAA & Formación & $\begin{array}{l}\text { Posición } \\
\text { Formación }\end{array}$ & Talento & $\begin{array}{l}\text { Posición } \\
\text { Talento }\end{array}$ & Total & $\begin{array}{l}\text { Posición } \\
\text { Total }\end{array}$ & N $^{\circ}$ Empresas \\
\hline Cantabria & 184.2 & 1 & 172.5 & 7 & 785.0 & 1 & 6 \\
Islas Baleares & 181.7 & 2 & 180.0 & 3 & 757.2 & 6 & 6 \\
País vasco & 180.0 & 3 & 175.8 & 6 & 771.6 & 3 & 12 \\
C. Valenciana & 178.3 & 4 & 153.3 & 11 & 718.7 & 7 & 3 \\
Cataluña & 176.2 & 5 & 176.6 & 5 & 775.5 & 2 & 51 \\
Madrid & 175.3 & 6 & 177.6 & 4 & 766.6 & 5 & 307 \\
Asturias & 167.5 & 7 & 157.5 & 10 & 692.0 & 12 & 2 \\
Andalucía & 166.3 & 8 & 161.3 & 9 & 718.0 & 8 & 4 \\
Islas Canarias & 160.0 & 9 & 185.0 & 2 & 710.0 & 11 & 1 \\
Aragón & 157.5 & 10 & 152.5 & 12 & 716.5 & 9 & 2 \\
Galicia & 156.7 & 11 & 188.3 & 1 & 771.0 & 4 & 3 \\
Murcia & 155.0 & 12 & 145.0 & 13 & 635.0 & 13 & 1 \\
Castilla y León & 142.5 & 13 & 165.5 & 8 & 711.0 & 10 & 2 \\
Total general & 175.2 & & 176.7 & & 765.8 & & 400 \\
Correlación & & & $23.8 \%$ & & & & \\
Pearson & & & & & & & \\
Formación- & & & & & & & \\
Talento & & & & & & & \\
\hline
\end{tabular}

Fuente: Elaboración propia a partir de los datos publicados en Actualidad Económica 2013-2016.

En la tabla 6 se pueden ver los valores promedios de la Formación y de la Gestión del Talento por áreas regionales en España, en la que apenas existen diferencias entre zonas, sin tener en cuenta la agrupación el "Resto" que ocupa la última posición y representa el menor número de casos. En cuanto al número de empresas, si se aprecia que predominan las empresas ubicadas en la zona centro de España, concretamente en Madrid que es la capital del país.

Tabla 6

La Formación y la Gestión del Talento de las empresas por áreas regionales

\begin{tabular}{|c|c|c|c|c|c|c|c|}
\hline $\begin{array}{l}\text { Agrupaciones } \\
\text { regionales }\end{array}$ & $\begin{array}{l}\text { Valor } \\
\text { promedio } \\
\text { Formación }\end{array}$ & $\begin{array}{l}\text { Posición } \\
\text { Formación }\end{array}$ & $\begin{array}{l}\quad \text { Valor } \\
\text { promedio } \\
\text { Talento }\end{array}$ & $\begin{array}{l}\text { Posición } \\
\text { Talento }\end{array}$ & $\begin{array}{l}\quad \text { Valor } \\
\text { promedio } \\
\text { Total }\end{array}$ & $\begin{array}{l}\text { Posición } \\
\text { Total }\end{array}$ & $\begin{array}{c}\mathrm{N}^{\mathrm{o}} \\
\text { Empresas }\end{array}$ \\
\hline Norte & 177.0 & 1 & 175.0 & 3 & 768.1 & 2 & 23 \\
\hline Mediterráneo & 176.5 & 2 & 175.2 & 2 & 768.6 & 1 & 61 \\
\hline Centro & 175.3 & 3 & 177.6 & 1 & 766.6 & 3 & 307 \\
\hline Resto & 158.3 & 4 & 162.9 & 4 & 715.2 & 4 & 9 \\
\hline Total general & 175.2 & & 176.7 & & 765.8 & & 400 \\
\hline
\end{tabular}

Fuente: Elaboración propia a partir de los datos publicados en Actualidad Económica 2013-2016. 
La formación y la gestión del talento según el tamaño de la empresa

Se aprecia en la tabla 7 como el $87 \%$ de las empresas tienen plantillas medias superiores a los 250 trabajadores. El factor Formación muestra unos resultados donde la media de valoración es mayor en las empresas más grandes (176.06) frente a las demás (169.47) y en este caso estadísticamente relevante.

En cambio, el comportamiento de la variable Gestión del Talento es distinto, pues las empresas de menor dimensión tienen una media de valoración más alta (180.88) que las grandes (176.14), sin ser significativas dichas diferencias. Por tanto, se puede concluir que el tamaño de las empresas no es relevante en la Gestión del Talento.

Tabla 7

Estadísticos y prueba de muestras independientes de la Formación, Talento, clasificadas por tamaños de empresas

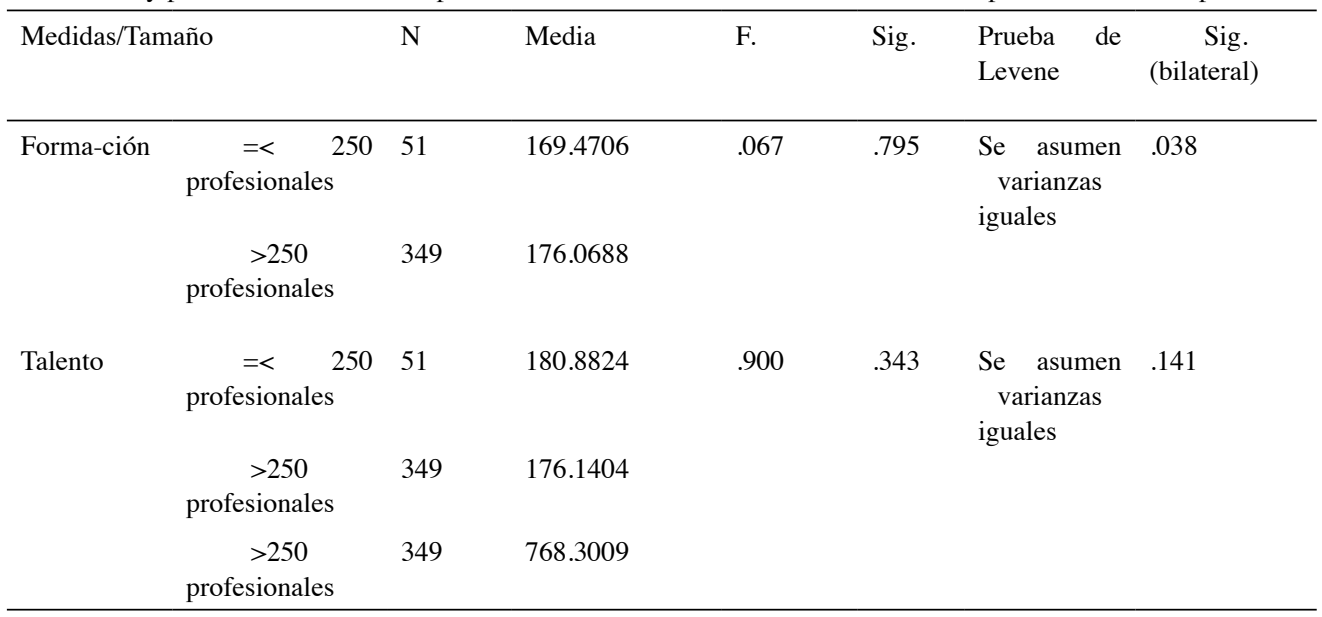

Fuente: Elaboración propia a partir de los datos publicados en Actualidad Económica 2013-2016.

\section{La formación y la gestión del talento según cotice la empresa en el mercado bursátil}

Se observa en la tabla 8 que el $76.25 \%$ de las empresas más atractivas para el desempeño profesional cotizan en el mercado bursátil. Los datos referidos a la Formación sí muestran que las empresas cotizantes en el mercado de valores tienen valores superiores (177.00) que las demás, pues su sig bilateral es $<0,05$; por tanto se puede considerar que estas medias son relevantes. Respecto a la Gestión del Talento, la sig bilateral es de 0.085 , valores muy próximos a la relevancia estadísticas a favor de las empresas que cotizan en bolsa con un valor promedio 178.03 puntos frente a los 174.09 de las otras. 
Tabla 8

Estadísticos y prueba de muestras independientes de la Formación, Talento, según cotización de las empresas

\begin{tabular}{|c|c|c|c|c|c|c|c|}
\hline \multicolumn{2}{|c|}{$\begin{array}{l}\text { Medidas/Cotización } \\
\text { Mercado Bursatil }\end{array}$} & $\mathrm{N}$ & Media & F. & Sig. & $\begin{array}{l}\text { Prueba de } \\
\text { Levene }\end{array}$ & Sig. (bilateral) \\
\hline \multirow[t]{2}{*}{ Forma-ción } & No cotiza & 131 & 171.5878 & 1.031 & .311 & $\begin{array}{l}\text { Se asumen } \\
\text { varianzas } \\
\text { iguales }\end{array}$ & .016 \\
\hline & Sí cotiza & 269 & 177.0000 & & & & \\
\hline \multirow[t]{2}{*}{ Talento } & No cotiza & 131 & 174.0992 & 1.172 & .280 & $\begin{array}{l}\text { Se asumen } \\
\text { varianzas } \\
\text { iguales }\end{array}$ & .085 \\
\hline & Sí cotiza & 269 & 178.0335 & & & & \\
\hline
\end{tabular}

Fuente: Elaboración propia a partir de los datos publicados en Actualidad Económica 2013-2016.

Análisis global de la formación y la gestión del talento

En este punto se estimará en qué medida las diferencias en los valores de las variables independientes modifican la valoración de la Formación de las empresas, de modo que se mantienen constantes las demás variables. Para ello, se estima un modelo de regresión lineal múltiple mediante mínimos cuadrados ordinarios para cada uno de los cuatro años (año $=$ modelo). En cada uno de los modelos la variable dependiente es la valoración del nivel formativo. Las variables independientes son:

1. El área geográfica española a la que pertenece la sede de la empresa, que es una variable categórica, se considera el área de referencia la Comunidad de Madrid.

2. El área geo-cultural del país de nacionalidad de la empresa, variable también categórica, se considera el grupo de referencia el de los países anglosajones.

3. La nacionalidad española de la empresa, variable dicotómica, se considera el grupo de referencia las empresas de nacionalidad no española.

4. El tamaño de la empresa, variable dicotómica con las categorías grande y pequeña, se considera el grupo de referencia el de las empresas pequeñas.

5. El número de trabajadores de la empresa en todo el mundo.

6. El número de trabajadores de la empresa en España.

7. La cotización en bolsa, variable dicotómica, se considera el grupo de referencia el de las empresas que no cotizan en bolsa.

8. El sector de actividad económica de la empresa según la categorización de la CNAE, se considera el grupo de referencia el sector agricultura, ganadería, silvicultura y pesca.

En todos los modelos, el número de observaciones es idéntico: las 100 empresas del ranking de la $R A E$ del año en cuestión. El Anexo I muestra los resultados de los cuatro modelos de regresión. El modelo para el año 2016 es estadísticamente significativo $(F(24,75)=1.7 ; p(F)$ $<.05)$; para todos los demás, $p(F)>.1)$.

La cotización en bolsa alcanza significación estadística (al nivel del .01) en al menos dos modelos (2014 y 2016). En ambos casos, las empresas que cotizan en bolsa tienen una mayor valoración, y aumenta el intercepto en 10.17 y 10.61 puntos, respectivamente. Otras variables no son estadísticamente significativas más que en alguno de los modelos: 
- Las Comunidades Autónomas lo son en el modelo del año 2016; la prueba anova para modelos anidados (es el modelo completo el estimado en este trabajo para cada año, y el modelo reducido el mismo modelo sin las tres variables dummy que representan a las Comunidades Autónomas agrupadas en tres conjuntos) arroja como resultado $F=$ $2.74448(p(F)<.05)$. Ello se debe al efecto de las comunidades del resto del país, que reducen el intercepto en 40.04 puntos. Al margen de la significación estadística, de esta variable sólo cabe señalar que las comunidades del Norte reducen el intercepto en los modelos de 2014, 2015 y 2016.

- La nacionalidad española lo es en el modelo de 2013, al nivel del 1\%, se aumenta el intercepto en 14.84 puntos. Además, el signo es positivo en tres de los tres modelos (2013, 2014 y 2016).

- El sector de actividad económica lo es en el modelo de 2015 (la prueba para modelos anidados -es el modelo completo el estimado y el modelo anidado aquel en el que eliminan las variables dummy representativas de los sectores de actividad arroja como resultado $F=1.90464, p(F)<.1$ ).

Al margen de la significación estadística de las variables, se detectan ciertas pautas o tendencias que en una investigación más sistemática cabría desentrañar. Por ejemplo, si se atiende al signo de los coeficientes, en tres de los modelos, la pertenencia de las empresas tanto al área geo-cultural mediterránea $(2014,2015$ y 2016) como al área del resto de países (2013, 2014 y 2015) parece aumentar su puntuación respecto a las empresas del área anglosajona, mientras que su pertenencia al área del Centro y Norte de Europa parece contribuir a reducir la valoración de las empresas (2013, 2015 y 2016).

$\mathrm{Si}$ se atiende al signo, las empresas grandes tienen mayores puntuaciones en los cuatro modelos; y la cotización en bolsa muestra una relación positiva creciente.

Si se centra la atención en el Talento, se estimará en qué medida modifica la valoración de dicha variable las diferencias en los valores de los factores independientes, si se mantienen constantes las demás variables. Para ello, se estima también un modelo de regresión lineal múltiple mediante mínimos cuadrados ordinarios para cada uno de los cuatro años; la valoración del talento es la variable dependiente en este caso. El Anexo II muestra los resultados de los cuatro modelos de regresión. El modelo para el año 2015 es estadísticamente significativo $(p(F)$ $<.05$ ); para todos los demás, $p(F)>.1$ ).

Dos variables independientes alcanzan significación estadística (al nivel del .05) en al menos dos modelos. La primera es el tamaño de la empresa, aunque el signo de su efecto en uno de los modelos es el opuesto al otro; así, en 2013, las empresas grandes son más valoradas que las pequeñas (se aumenta el valor del intercepto en 16.17 puntos), mientras que en 2016 son menos valoradas (se reduce el valor del intercepto en 17.11 puntos). La segunda de las variables es la cotización en bolsa. En ambos modelos (años 2013 y 2015), las empresas que cotizan en bolsa tienen una mayor valoración que aquellas que no cotizan, aumentan el intercepto en 11.35 y 12.21 puntos, respectivamente.

A pesar de que en el modelo del año 2013 la categoría de los Países Mediterráneos tiene significación estadística, no cabe otorgar un excesivo valor al resultado, puesto que el área geocultural de la nación de la empresa no tiene significación estadística.

El sector de actividad económica tiene significación estadística en los modelos de los años 2013 y 2015. 
Se detectan en el Anexo II ciertas pautas o tendencias como, por ejemplo, si se atiende al signo de los coeficientes, en tres de los modelos la pertenencia de las empresas tanto al área geocultural norte y centro-europea $(2014,2015$ y 2016) como al área mediterránea $(2013,2014$ y 2015) parece reducir su puntuación respecto a las empresas del área anglosajona, mientras que su pertenencia a otros países parece contribuir a aumentar el atractivo de las empresas.

La nacionalidad española de la empresa (estadísticamente significativa al nivel del 0.5 en el modelo de 2013) tiene signo positivo en todos los modelos, menos en el de 2014. La variable cotización en bolsa tiene signo positivo en los modelos de 2013, 2015 y 2016: cotizar en bolsa aumenta el atractivo de las empresas.

\section{Limitaciones}

Este artículo revela una serie de limitaciones con relación al objetivo establecido. En primer lugar, es un trabajo cuantitativo limitado a dos variables independientes (Formación y Gestión del Talento) que valora el atractivo de la empresa para el desempeño laboral. Otra limitación fue la debida a centrarse en añadir otras variables objetivas, tales como sector, nacionalidad, tamaño, cotización bursátil o ubicación regional, mientras se evitan factores como el liderazgo, capacidad de gestión, variables de carácter cualitativo y cuya valoración es más compleja. Otra limitación es temporal, ya que son datos para un período concreto comprendido entre los años 2013 y 2016. Esto se une a la limitación nacional pues se centran en las empresas que operan en un país europeo.

\section{Discusión y conclusiones}

El objetivo del artículo es analizar el perfil de las empresas más excelentes para trabajar en España a través de las variables Formación y Gestión del Talento cuando se usan como factores de análisis la actividad empresarial, nacionalidad, ubicación regional, tamaño y cotización en bolsa, así como su posible relevancia estadística.

Se observa que en la variable Formación las empresas más valoradas se dedican a actividades sanitarias y de servicios sociales; y las profesionales y científicas, la cual también sale como valor destacado en la Gestión del Talento. El hecho de tener una correlación tan baja entre las variables Formación-Gestión del Talento (12.2\%), indica que no tienen comportamientos paralelos. El Anexo I y II muestran que el sector de actividad económica es significativo para el año 2015 en la variable Formación, y el 2013 y 2015 para la variable Gestión del Talento en algunos sectores, pero no se puede concluir que la actividad económica tenga relevancia estadística, lo que va en contra de los estudios de Jackson y Schuler (1995, pp. 251 y ss.) que sí lo consideraban un factor contextual en la práctica de recursos humanos. Destaca que el estudio se refiere a dos variables concretas como son la Formación y la Gestión del Talento para las empresas que figuran en el ranking de las cien empresas más atractivas para trabajar publicado por la $R A E$, y en cierta medida tiene lógica que no existan diferencias ya que se trata, según dicho ranking, de las mejores empresas y entre estas no tienen por qué manifestarse disparidades significativas.

La mayor relación entre la Formación y el Talento se produce al analizarlo por países, pues alcanza un 43.4\%, lo que explica que las naciones con mayor valoración en Formación sean las empresas de Corea del Sur y Suiza que también están entre las primeras posiciones al analizar 
los valores de Gestión del Talento. España es el país con mayor número de empresas (38.7\%) seguida de EEUU. Al agruparlo por áreas internacionales, el mayor número provienen de Europa Mediterránea y las de mayor valoración son las anglosajonas. Destaca la baja valoración de las empresas del Norte y Centro de Europa. A pesar de que los análisis estadísticos muestran años y áreas geográficas con significación estadística, tampoco se manifiesta en términos globales que la nacionalidad de las empresas tenga influencia relevante en las variables objeto de estudio, lo cual va en consonancia con lo concluido por Ibrahim y Shah (2013) y no con los estudios de Ferner (1997); Liu (2004); y Guthrie et al. (2008). La no relevancia estadística muestra un hallazgo importante, ya que se podría esperar que empresas de países anglosajones y de Europa Centro-Norte (zonas de mayor desarrollo económico) mostraran mejores resultados que las de Europa Mediterránea, que es la de mayor valoración en la variable Formación.

Al centrarnos en el eje de análisis ubicación de su sede central la correlación FormaciónTalento es del 23.8\%, y que supera tan sólo a la que se obtenía por sectores económicos. Esto explica que las empresas de Cantabria, Islas Baleares y País Vasco son líderes en Formación; en cambio en Gestión del Talento, aparece en primer lugar Galicia (Inditex), seguida de las Islas Canarias. En cuanto al número de empresas, éstas se concentran en Madrid (76.5\%), como capital del país; aspecto lógico al ser grandes empresas que operan en todo el territorio nacional e internacional. Cataluña es la segunda comunidad con mayor número de organizaciones (12.75\%), y es la comunidad con mayor PIB de España. Por áreas geográficas no existen diferencias apreciables y tampoco la ubicación tiene influencia significativa en el valor de estas variables. Aunque en algún año la ubicación se muestra de manera significativa (anexos I y II), en líneas generales podemos concluir que no es relevante, lo que indica una información muy valiosa como que las empresas de las regiones españolas con mayor desarrollo económico, como son Madrid, Cataluña o el País Vasco, tengan una menor valoración media en la gestión de las variables Formación y Gestión del Talento que las de regiones menos desarrolladas como Galicia o Cantabria.

Al analizar el perfil según el tamaño son mayores los valores medios de las empresas con más de 250 trabajadores por compañía, significativas para la Formación, no así para la Gestión del Talento. El que el tamaño de las empresas influya significativamente en la variable Formación va en línea con los estudios de Ibrahim y Shah (2013, pp. 7, 14 y ss.) y manifiesta una lógica en la medida que las empresas más grandes tienen mayores recursos y mayor capacidad organizativa para llevar a cabo planes de formación para sus empleados. También resulta clarificador concluir que la dimensión de la empresa no afecta a la Gestión del Talento, ya que no había ningún estudio que hiciera pensar en una hipótesis en dicha dirección.

Lo mismo se puede concluir al relacionarlo con la cotización bursátil de las empresas, las que cotizan tienen mayores valores medios relevantes en la Formación. En el caso el Talento presenta una sig bilateral del 0.085 (tabla 8 ) valor muy cercano a la significatividad. Se puede concluir que las empresas que cotizan en bolsa presentan mejores resultados en ambas variables y relevantes, lo cual constituye un hallazgo novedoso.

Como conclusión final, decir que se aprecia un comportamiento dispar en las variables vinculadas al conocimiento, Formación y la Gestión del Talento, si bien se puede concluir que las empresas que cotizan en bolsa y en menor medida el tamaño muestran relaciones positivas con las variables objeto de estudio, y en cambio la nacionalidad, la ubicación y la actividad de las empresas no tienen influencia estadísticamente relevante en el valor de la Formación y la Gestión del Talento. 
Futuras investigaciones deberían identificar otras variables omitidas y contrastar si la cotización en el mercado bursátil y el tamaño reflejarán igualmente una cierta influencia en las valoraciones de la Formación y la Gestión del Talento para las empresas con mayor excelencia en Recursos Humanos en España. Otra línea de investigación podría ser analizar este modelo en otros países del mundo.

\section{Referencias}

Amossé, T., Bryson, A., Forth, J. y Petit, H. (2016). Managing and working in Britain and France: An introduction. En T. Amossé, A. Bryson, J. Forth y H. Petit (Eds.), Comparative workplace employment relations, An analysis of practice in Britain and France. (pp. 1-26). Londres: Palgrave MacMillan. https://doi.org/10.1057/978-1-13757419-0_1

Ballou, B., Godwin, N. H. y Shortridge, R. T. (2003). Firm value and employee attitudes on workplace quality. Accounting Horizons, 17 (4), 329-341. https://doi.org/10.2308/acch.2003.17.4.329

Baron, R. (1991). Motivation in work settings: Reflections on the core of organizational research. Motivation and Emotion, 15 (1), 1-8. https://doi.org/10.1007/BF00991472

Betcherman, G.; Leckie, N. and McMullen, K. (1997). Developing Skills in the Canadian Workplace: The Results of the EKOS Workplace Training Survey. Otawa: Canadian Policy Research Networks.

Bonache Pérez, J. (1996). El papel de la política de recursos humanos en la internacionalización de la empresa. Economía Industri, (307), 37-48.

Del Campo, M.O. y Salcines, V. (2008). El valor económico de la Educación a través del pensamiento económico en el siglo XX. Revista de la Educación Superior, vol. XXXVII, (147), 45-61. https://doi.org/10.1016/j.resu.2016.08.001

Conway, N., Deakin, S., Komzelmann, S., Petit, H., Rebérioux, A. y Wilkinson, F. (2008). The influence of stock market listing on human resource management: Evidence for France and Britain. British Journal of Industrial Relations, 46 (4), 632-673. https://doi.org/10.1111/j.1467-8543.2008.00698.x

Ferner, A. (1997). Country of origin effects and HRM in multinational companies. Human Resource Management Journal, 7 (1), 19-37. https://doi.org/10.1111/j.1748-8583.1997.tb00271.x

Fields, D., Chan, A. y Akhtar, S. (2002). Organizational context and human resource management strategy: A structural equation analysis of Hong Kong firms. International Journal of Human Resource Management, 11 (2), $264-277$. https://doi.org/10.1080/095851900339864

Finegold, D. and Sosckice, D. (1988). The Failure of British Training: Analysis and Prescription. Oxford Review of Economic Policy, (4), 21-53. https://doi.org/10.1093/oxrep/4.3.21

Friedman, R. (2014). The best place to work. The art and science of creating an extraordinary workplace. Nueva York: Penguin.

Fulmer, I. S., Gerhart, B. y Scott, K. S. (2003). Are the 100 Best better? An empirical investigation of the relationship between being a "Great Place to Work" and firm performance. Personnel Psychology, 56 (4), 965-993. https://doi. org/10.1111/j.1744-6570.2003.tb00246.x

Gallie, D. (2007). Production regimes and the quality of employment in Europe. Annual Review of Sociology, 33 (1), 85-104. https://doi.org/10.1146/annurev.soc.33.040406.131724

Gallie, D. (2009). Production regimes, employment regimes and the quality of work. En D. Gallie (Ed.), Employment regimes and the quality of work. (pp. 1-33). Nueva York: Oxford Univerity Press. https://doi.org/10.1093/acprof:oso/9780199230105.001.0001

Grimshaw, D. y Rubery, J. (2007). Economics and HRM. En P. Boxall, J. Purcell y P. Wright (Eds.), The Oxford Handbook of Human Resource Management. (pp. 68-87). Nueva York: Oxford Univerity Press. https://doi.org/10.1111/j.1468-2338.2007.00475.x

Guest, D. E. (2007). HRM and the worker: Towards a new psychological contract? En P. Boxall, J. Purcell y P. Wright (Eds.), The Oxford Handbook of Human Resource Management. (pp. 128-146). Oxford: Oxford Univerity Press.

Guinot, J., Chiva, R. y Mallén, F. (2015). Altruismo y capacidad de aprendizaje organizativo: Un estudio en las empresas mejor valoradas por los trabajadores en España. Universia Business Review, (45), 92-109. 
Guthrie, J. P., Liu, W., Flood, P. C. y MacCurtain, S. (2008). High performance work systems, workforce productivity, and innovation: A comparison of MNCs and indigenous firms (WP 04-08). Dublín: DCU Business school. doras.dcu. ie/2421/1/wp0408.pdf

Hall, R. (1992). The strategic analysis of intangible resources. Strategic Management Joournal, (13), 145-158. https://doi. org/10.1002/smj.4250130205

Hinkin, T. R. y Tracey, J. B. (2010). What makes it so great? An analysis of human resources practices among Fortune's Best Companies to Work for. Cornell Hospitality Quarterly, 51 (2), 158-170. https://doi.org/10.1177/1938965510362487

Hodulak, M. (2017). Global corporate workplace. Implementing new global workplace standards in a local context. Munich: Springer. https://doi.org/10.1007/978-3-662-53392-5

Hosie, P., Jayashree, P., Tchantchane, A. and Ban Seng Lee (2013). The effect of autonomy, training opportunities, age and salaries on job satisfaction in the South East Asian retail petroleum industry. The International Journal of Human Resource Management, 24 (21), 3980-4007. https://doi.org/10.1080/09585192.2013.829517

Ibrahim, H. I. y Shah, K. A. M. (2013). Effects of organizational characteristics factors on the implementation of strategic human resource practices: Evidence from malaysian manufacturing firms. ECONOMIA. Seria MANAGEMENT, 16 (1), 5-24.

Jackson, S. y Schuler, R. S. (1995). Understanding human resource management in the context of organizations and their environments. Annual Review of Psychology, 46, 237-264. https://doi.org/10.1146/annurev.ps.46.020195.001321

Joo, B. K. y McLean, G. N. (2006). Best employer studies: A conceptual model from a literature review and a case study. Human Resource Development Review, 5 (2), 228-257. https://doi.org/10.1177/1534484306287515

Joyce, K. E. (2003). Lessons for employers from Fortune's "100 best”. Business Horizons, 46 (2), 77-84. https://doi. org/10.1016/S0007-6813(03)00013-2

Kok, J. de y Uhlaner, L. M. (2001). Organization context and human resource management in the small firm. Small Business Economics, 17 (4), 273-291. https://doi.org/10.1023/A:1012238224409

Kortekaas, F. (2007). HRM, organizational performance and the role of firm size, Master's thesis, Erasmus School of Economics, Rotterdam. https://doi.org/10.5430/mos.v2n3p17

Lenaghan, J. A. y Eisner, A. B. (2006). Employers of choice and competitive advantage: The proof of the pudding is in the eating. Journal of Organizational Culture, Communications and Conflict, 10 (1), 99-109.

Liu, W. (2004). The cross national transfer of HRM practices in MNCs: An integrative research model. International Journal of Manpower, 25 (6), 500-517. https://doi.org/10.1108/01437720410560415

Mincer, J. (1962). On the Job Training: Costs, Returns, and Some Implications. Journal of Political Economy, 70 (5), 50-79. https://doi.org/10.1086/258725

Morgan, J. (2014). The future of work. Attract new talent, build better leaders, and create a competitive organization. Hoboken, NJ: Wiley.

Nijhof, W., De-Jong, M., and Beukhof, G. (1998). Employee commitment in changing organizations: an exploration. Journal of European Industrial Training, 22 (6), 243-248. https://doi.org/10.1108/03090599810224701

OCDE (1991). Employment Outlook. Julio, OCDE, Paris.

Osterman, P. (1994). How Common Is Workplace Transformation and Who Adopts It? Industrial and Labor Relations Review, 47 (2), 173-188. https://doi.org/10.1177/001979399404700202

Paawee, J. y Boselie, P. (2007). HRM and societal embeddedness. En P. Boxall, J. Purcell y P. Wright (Eds.), The Oxford Handbook of Human Resource Management. (pp. 166-184). Oxford: Oxford Univerity Press. https://doi.org/10.1093/ oxfordhb/9780199547029.001.0001

Quintanilla, J. y Ferner, A. (2003). Multinationals and human resource management: Between global convergence and national identity. The International Journal of Human Resource Management, 14 (3), 363-368. https://doi. org/10.1080/0958519022000031799

Romero, E. J. (2004). Are the great places to work also great performers? Academy of Management Perspectives, 18 (2), 150-152. https://doi.org/10.5465/AME.2004.13835923

Smith, A. (1993). Training and Enterprise Performance: Is There any Link? Training Agenda, 1 (3), 24-27.

Wright, P.M.; McMahan, G.C. and McWilliams, A. (1994). Human resources and sustained competitive advantage: a resource-based perspective. International Journal of Human Resource Management, (5), 301-326. https://doi. org/10.1080/09585199400000020 


\section{Anexo I}

Modelo de regresión lineal múltiple con el valor de la Formación de las empresas como variable dependiente en el período 2013-2016

\begin{tabular}{|c|c|c|c|c|}
\hline & 2013 & 2014 & 2015 & 2016 \\
\hline \multirow[t]{2}{*}{ Intercepto } & $165.29^{* * *}$ & $121.47^{* * *}$ & $156.96^{* * *}$ & $157.94^{* * *}$ \\
\hline & $(15.14)$ & $(26.74)$ & $(13.23)$ & $(18.05)$ \\
\hline \multirow[t]{2}{*}{ CCAA Mediterráneo } & 2.03 & -5.46 & -2.34 & 6.66 \\
\hline & $(6.48)$ & $(5.99)$ & $(5.22)$ & $(7.70)$ \\
\hline \multirow[t]{2}{*}{ CCAA Norte } & 4.26 & -4.69 & -3.53 & -8.11 \\
\hline & $(9.31)$ & (11.47) & $(10.63)$ & (11.91) \\
\hline \multirow[t]{2}{*}{ CCAA resto } & & -3.42 & 16.28 & $-40.04^{* *}$ \\
\hline & & $(14.56)$ & $(10.40)$ & $(15.83)$ \\
\hline \multirow[t]{2}{*}{ Resto de países } & 8.70 & 22.83 & 10.46 & -4.97 \\
\hline & $(12.92)$ & $(15.03)$ & $(13.23)$ & (19.38) \\
\hline \multirow[t]{2}{*}{ Países Centro y Norte Europa } & -1.13 & 2.10 & -4.80 & -7.74 \\
\hline & $(6.37)$ & $(6.18)$ & $(5.40)$ & $(8.15)$ \\
\hline \multirow[t]{2}{*}{ Países Mediterráneos Europa } & -10.00 & .71 & 7.90 & 2.22 \\
\hline & $(7.58)$ & $(7.34)$ & $(6.29)$ & $(9.69)$ \\
\hline \multirow[t]{2}{*}{ Nacionalidad española } & $14.84^{*}$ & 3.38 & -6.81 & 7.34 \\
\hline & $(8.25)$ & $(7.77)$ & $(6.70)$ & $(8.87)$ \\
\hline \multirow[t]{2}{*}{ Tamaño } & 6.22 & 6.34 & 3.77 & 2.60 \\
\hline & $(6.75)$ & $(6.86)$ & $(6.04)$ & $(8.50)$ \\
\hline \multirow[t]{2}{*}{ Número de trabajadores (Mundo) } & 0.00 & 0.00 & 0.00 & 0.00 \\
\hline & $(0.00)$ & $(0.00)$ & $(0.00)$ & $(0.00)$ \\
\hline \multirow[t]{2}{*}{ Número de trabajadores (España) } & -0.00 & 0.00 & 0.00 & -0.00 \\
\hline & $(0.00)$ & $(0.00)$ & $(0.00)$ & $(0.00)$ \\
\hline \multirow[t]{2}{*}{ Cotización en bolsa } & 2.63 & $10.17^{*}$ & 7.63 & $10.61^{*}$ \\
\hline & $(5.78)$ & $(5.46)$ & $(4.81)$ & $(6.09)$ \\
\hline \multirow[t]{2}{*}{ CNAE Industria manufacturera } & & 28.67 & & \\
\hline & & $(23.91)$ & & \\
\hline \multirow[t]{2}{*}{$\begin{array}{l}\text { CNAE Suministro de energía eléctrica, gas, } \\
\text { vapor y aire acondicionado }\end{array}$} & -14.49 & 31.12 & $17.93^{*}$ & 10.66 \\
\hline & $(11.28)$ & $(26.04)$ & $(9.83)$ & $(15.92)$ \\
\hline \multirow[t]{2}{*}{ CNAE Construcción } & 34.23 & 42.47 & 10.25 & -8.89 \\
\hline & $(22.19)$ & $(31.39)$ & $(13.53)$ & $(17.12)$ \\
\hline \multirow{2}{*}{$\begin{array}{l}\text { CNAE Comercio al por mayor y al por } \\
\text { menor; reparación de vehículos de motor y } \\
\text { motocicletas }\end{array}$} & -10.77 & 16.57 & 1.98 & -10.04 \\
\hline & $(8.42)$ & $(24.96)$ & $(7.01)$ & $(11.43)$ \\
\hline \multirow[t]{2}{*}{ CNAE Transporte y almacenamiento } & 21.96 & 39.05 & -15.81 & 32.43 \\
\hline & $(16.22)$ & $(25.97)$ & $(11.75)$ & (19.87) \\
\hline \multirow[t]{2}{*}{ CNAE Hostelería } & 4.63 & 26.49 & 8.94 & -3.54 \\
\hline & $(17.32)$ & $(26.63)$ & (11.92) & $(28.31)$ \\
\hline \multirow[t]{2}{*}{ CNAE Edición } & -9.22 & 25.06 & -4.29 & -15.66 \\
\hline & $(8.46)$ & $(24.63)$ & $(8.08)$ & $(12.61)$ \\
\hline \multirow[t]{2}{*}{ CNAE Actividades financieras y de seguros } & -3.96 & 34.83 & 6.45 & -3.08 \\
\hline & $(7.63)$ & $(24.51)$ & $(6.61)$ & $(10.28)$ \\
\hline CNAE Actividades inmobiliarias & -14.61 & 33.36 & 15.00 & -16.67 \\
\hline
\end{tabular}




\begin{tabular}{|c|c|c|c|c|}
\hline & 2013 & 2014 & 2015 & 2016 \\
\hline & (22.14) & $(27.50)$ & (13.45) & $(20.45)$ \\
\hline \multirow[t]{2}{*}{$\begin{array}{l}\text { CNAE Actividades profesionales, científicas } \\
\text { y técnicas }\end{array}$} & 5.37 & 34.94 & $14.18^{*}$ & 13.57 \\
\hline & $(8.14)$ & (23.73) & $(7.23)$ & $(10.77)$ \\
\hline \multirow[t]{2}{*}{$\begin{array}{l}\text { CNAE Actividades administrativas y } \\
\text { servicios auxiliares }\end{array}$} & -13.57 & 13.36 & $21.96^{* *}$ & 5.48 \\
\hline & (12.12) & $(25.29)$ & $(9.64)$ & (12.92) \\
\hline \multirow[t]{2}{*}{$\begin{array}{l}\text { CNAE Administración pública y defensa; } \\
\text { seguridad social obligatoria }\end{array}$} & & & & -18.41 \\
\hline & & & & $(26.94)$ \\
\hline \multirow[t]{2}{*}{ CNAE Educación } & & $65.08^{* *}$ & & -22.60 \\
\hline & & (31.32) & & (26.07) \\
\hline \multirow[t]{2}{*}{$\begin{array}{l}\text { CNAE Actividades sanitarias y de servicios } \\
\text { sociales }\end{array}$} & 12.04 & 43.48 & 11.56 & 3.57 \\
\hline & $(21.41)$ & $(26.42)$ & (13.38) & $(26.15)$ \\
\hline Observaciones & 100 & 100 & 100 & 100 \\
\hline$R^{2}$ & .20 & .29 & .29 & .35 \\
\hline$R^{2}$ Ajustado & -.01 & .06 & .09 & .15 \\
\hline Error típico residual & $20.27(\mathrm{gl}=78)$ & $19.06(\mathrm{gl}=75)$ & $16.92(\mathrm{gl}=77)$ & $24.13(\mathrm{gl}=75)$ \\
\hline$F$ & $.96(\mathrm{gl}=21 ; 78)$ & $1.25(\mathrm{gl}=24 ; 75)$ & $1.43(\mathrm{gl}=22 ; 77)$ & $1.70 * *(\mathrm{gl}=24 ; 75)$ \\
\hline Notas: & $\begin{array}{l}* * * p<.01 ; * * p \\
<.05 ; * p<.1\end{array}$ & & & \\
\hline
\end{tabular}

\section{Anexo II}

Modelo de regresión lineal múltiple con el valor del Talento de las empresas como variable dependiente en el período 2013-2016.

\begin{tabular}{|c|c|c|c|c|}
\hline & 2013 & 2014 & 2015 & 2016 \\
\hline \multirow[t]{2}{*}{ Intercepto } & $126.98^{* * *}$ & $207.45^{* * * *}$ & $178.72^{* * *}$ & $207.61^{* * *}$ \\
\hline & (13.77) & $(27.92)$ & $(13.52)$ & (17.83) \\
\hline \multirow[t]{2}{*}{ CCAA Mediterráneo } & -1.76 & -2.38 & 2.00 & .88 \\
\hline & $(5.89)$ & $(6.26)$ & $(5.33)$ & $(7.61)$ \\
\hline \multirow[t]{2}{*}{ CCAA Norte } & -3.74 & 4.45 & $20.90^{*}$ & -7.65 \\
\hline & $(8.47)$ & (11.98) & $(10.86)$ & $(11.76)$ \\
\hline \multirow[t]{2}{*}{ CCAA resto } & & -5.61 & -11.78 & -4.57 \\
\hline & & $(15.20)$ & $(10.62)$ & $(15.64)$ \\
\hline \multirow[t]{2}{*}{ Resto de países } & 1.60 & 18.90 & 5.86 & 13.10 \\
\hline & (11.76) & $(15.70)$ & $(13.51)$ & (19.14) \\
\hline \multirow[t]{2}{*}{ Países Centro y Norte Europa } & 2.10 & -7.13 & -1.18 & -5.22 \\
\hline & $(5.79)$ & $(6.46)$ & $(5.52)$ & $(8.05)$ \\
\hline \multirow[t]{2}{*}{ Países Mediterráneos Europa } & $-12.66^{*}$ & -12.63 & -9.91 & .22 \\
\hline & $(6.89)$ & $(7.67)$ & $(6.42)$ & $(9.57)$ \\
\hline \multirow[t]{2}{*}{ Nacionalidad española } & $14.26^{*}$ & -1.33 & 9.64 & .08 \\
\hline & $(7.50)$ & $(8.11)$ & $(6.85)$ & $(8.76)$ \\
\hline \multirow[t]{2}{*}{ Tamaño } & $16.17^{* *}$ & -9.12 & -5.62 & $-17.11^{* *}$ \\
\hline & $(6.14)$ & $(7.16)$ & $(6.17)$ & $(8.40)$ \\
\hline \multirow[t]{2}{*}{ Número de trabajadores (Mundo) } & -0.00 & 0.00 & -0.00 & 0.00 \\
\hline & $(0.00)$ & $(0.00)$ & $(0.00)$ & $(0.00)$ \\
\hline
\end{tabular}




\begin{tabular}{|c|c|c|c|c|}
\hline & 2013 & 2014 & 2015 & 2016 \\
\hline \multirow[t]{2}{*}{ Número de trabajadores (España) } & -0.00 & 0.00 & -0.00 & -0.00 \\
\hline & $(0.00)$ & $(0.00)$ & $(0.00)$ & $(0.00)$ \\
\hline \multirow[t]{2}{*}{ Cotización en bolsa } & $11.35^{* *}$ & -2.36 & $12.21^{* *}$ & 8.82 \\
\hline & $(5.26)$ & $(5.70)$ & $(4.91)$ & $(6.01)$ \\
\hline \multirow[t]{2}{*}{ CNAE Industria manufacturera } & & -19.44 & & \\
\hline & & $(24.97)$ & & \\
\hline \multicolumn{5}{|l|}{ CNAE Suministro de energía } \\
\hline \multirow{2}{*}{$\begin{array}{l}\text { eléctrica, gas, vapor y aire } \\
\text { acondicionado }\end{array}$} & -3.13 & -4.19 & 11.57 & 3.67 \\
\hline & $(10.27)$ & (27.19) & $(10.04)$ & $(15.72)$ \\
\hline \multirow[t]{2}{*}{ CNAE Construcción } & 28.06 & 10.26 & 21.82 & -16.28 \\
\hline & (20.19) & (32.78) & $(13.82)$ & $(16.91)$ \\
\hline \multirow{2}{*}{$\begin{array}{l}\text { CNAE Comercio al por mayor y al } \\
\text { por menor; reparación de vehículos } \\
\text { de motor y motocicletas }\end{array}$} & $14.59^{*}$ & -13.17 & 6.15 & -17.18 \\
\hline & $(7.66)$ & $(26.07)$ & $(7.16)$ & $(11.29)$ \\
\hline \multirow[t]{2}{*}{ CNAE Transporte y almacenamiento } & 1.80 & -16.36 & -11.51 & $33.56^{*}$ \\
\hline & $(14.76)$ & $(27.12)$ & $(12.00)$ & (19.63) \\
\hline \multirow[t]{2}{*}{ CNAE Hostelería } & 8.79 & -9.24 & 17.91 & -17.26 \\
\hline & $(15.75)$ & $(27.81)$ & $(12.18)$ & $(27.96)$ \\
\hline \multirow[t]{2}{*}{ CNAE Edición } & 8.20 & -13.16 & $15.59^{*}$ & -11.96 \\
\hline & $(7.69)$ & $(25.72)$ & $(8.26)$ & $(12.45)$ \\
\hline \multirow{2}{*}{$\begin{array}{l}\text { CNAE Actividades financieras y de } \\
\text { seguros }\end{array}$} & $13.81^{*}$ & -9.90 & 8.67 & -4.03 \\
\hline & $(6.94)$ & $(25.59)$ & $(6.76)$ & $(10.15)$ \\
\hline \multirow[t]{2}{*}{ CNAE Actividades inmobiliarias } & 16.13 & -3.29 & 4.19 & -23.55 \\
\hline & (20.14) & (28.72) & (13.74) & $(20.20)$ \\
\hline \multirow{2}{*}{$\begin{array}{l}\text { CNAE Actividades profesionales, } \\
\text { científicas y técnicas }\end{array}$} & $27.94^{* * *}$ & -3.28 & $26.51^{* * *}$ & 8.83 \\
\hline & $(7.41)$ & $(24.78)$ & (7.39) & (10.64) \\
\hline \multirow[t]{2}{*}{$\begin{array}{l}\text { CNAE Actividades administrativas y } \\
\text { servicios auxiliares }\end{array}$} & 15.08 & -4.04 & $22.35^{* *}$ & -6.67 \\
\hline & (11.03) & $(26.41)$ & $(9.84)$ & (12.76) \\
\hline \multirow{2}{*}{$\begin{array}{l}\text { CNAE Administración pública y } \\
\text { defensa; seguridad social obligatoria }\end{array}$} & & & & -8.79 \\
\hline & & & & $(26.62)$ \\
\hline \multirow[t]{2}{*}{ CNAE Educación } & & $-71.21^{* *}$ & & -38.59 \\
\hline & & $(32.71)$ & & $(25.76)$ \\
\hline \multirow[t]{2}{*}{$\begin{array}{l}\text { CNAE Actividades sanitarias y de } \\
\text { servicios sociales }\end{array}$} & -9.88 & -14.82 & 11.29 & -14.76 \\
\hline & (19.47) & (27.59) & $(13.67)$ & $(25.83)$ \\
\hline Observaciones & 100 & 100 & 100 & 100 \\
\hline$R^{2}$ & .27 & .28 & .34 & .30 \\
\hline$R^{2}$ Ajustado & .07 & .05 & .15 & .07 \\
\hline Error típico residual & $18.44(\mathrm{gl}=78)$ & $19.90(\mathrm{gl}=75)$ & $17.28(\mathrm{gl}=77)$ & $23.84(\mathrm{gl}=75)$ \\
\hline$F$ & $1.37(\mathrm{gl}=21 ; 78)$ & $1.23(\mathrm{gl}=24 ; 75)$ & $1.82^{* *}(\mathrm{gl}=22 ; 77)$ & $1.32(\mathrm{gl}=24 ; 75)$ \\
\hline Notas: & \multicolumn{4}{|c|}{$* * * p<.01 ; * * p<.05 ; * p<.1$} \\
\hline
\end{tabular}

